\title{
Assessment and Validation of Collision "Consequence" Method of Assessing Orbital Regime Risk Posed by Potential Satellite Conjunctions
}

\author{
Travis F. Lechtenberga ${ }^{\mathrm{a}}$, and Matthew D. Hejduk ${ }^{\mathrm{b} *}$ \\ ${ }^{a}$ Conjunction Assessment Analyst, Omitron Inc., Colorado Springs, CO, USA \\ ${ }^{b}$ Astrorum Consulting LLC, Woodway, TX USA
}

\begin{abstract}
Collision risk management theory requires a thorough assessment of both the likelihood and consequence of potential collision events. Satellite conjunction risk assessment has produced a highly-developed theory for assessing the likelihood of collision but typically neglects to account for the consequences of a given collision. While any collision may compromise the operational survival of a space-craft, the amount of debris produced by the potential collision, and therefore the degree to which the orbital corridor may be compromised, can vary greatly among satellite conjunctions. Previous studies leveraged work on satellite collision modeling to develop a method to estimate whether a particular collision is likely to produce a relatively large or relatively small amount of resultant debris. The approximation of the number of debris pieces is dependent on a mass estimation process for the secondary objects utilizing the radar cross section of said object. This study examines the validity of the mass estimation process and establishes uncertainty bounds on the secondary object mass which will be used to best approximate the possible consequences of a prospective collision. This process is then applied to a large set of historical conjunctions to assess the frequency at which possible collisions may significantly augment the orbital debris environment in operational spacecraft.
\end{abstract} COEFFICIENTS; RISK MANAGEMENT

\begin{tabular}{|ll}
\hline Nomenclature \\
$A$ & Satellite Frontal Area $\left(\mathrm{m}^{2}\right)$ \\
$B C$ & Inverse Ballistic Coefficient $\left(\mathrm{m}^{2} / \mathrm{kg}\right)$ \\
$C_{d}$ & Drag Coefficient (dimensionless) \\
$C D M$ & Conjunction Data Message \\
$L_{c}$ & Characteristic Length $(\mathrm{m})$ \\
$M_{p}$ & Mass of Primary Object $(\mathrm{kg})$ \\
$M_{s}$ & Mass of Secondary Object $(\mathrm{kg})$ \\
$P_{c}$ & Probability of Collision \\
$R C S$ & Radar Cross Section $(\mathrm{m})$ \\
$S E M$ & Size Estimation Model \\
$S N R$ & Signal to Noise Ratio $($ dimensionless) \\
$\vec{v}$ & Satellite Velocity Vector (m/s) \\
$V_{r e l}$ & Relative Velocity (m/s) \\
$x$ & Longest Dimension of Object (m) \\
\hline
\end{tabular}

* Corresponding author. Tel.: 1-785-766-3943.

E-mail address: travis.lechtenberg@omitron.com. 


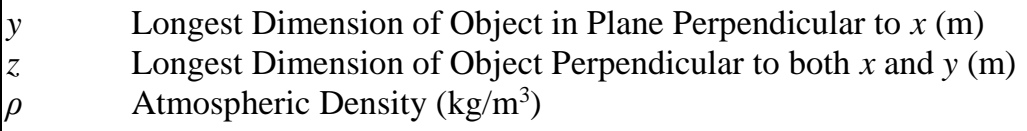

\section{Introduction}

In most current assessments of on orbit collision risk, operators typically are concerned only with the probability of collision $\left(P_{c}\right)$. This approach has existed since the early 1980's with collision likelihood methodology initially developed by Kaplan and Garrick [1]. As such, operators tend to view all likely conjunctions as equally disastrous, with a single possible outcome from a collision, primary object inoperability. This approach may become untenable as potential conjunction events increase in rate as additional spacecraft tracking capabilities come online and additional debris creation events occur. The orbital debris environment has seen large increases in population recently due to several events, including the Chinese ASAT test in 2007 against the FY-1C Fengyun satellite, the Iridium-Cosmos collision in 2009, and the projected increase in tracked debris population due to LockheedMartin's Space Fence, S-Band radar capabilities coming online in the near future.

As the spacecraft operational environment becomes more densely populated, consideration should be given to the potential consequences of a prospective collision. Potential collisions may be characterized as either catastrophic or non-catastrophic depending on the relative velocities of two objects and their masses. A catastrophic collision is one in which the primary object and the secondary object are fully fragmented and hence contributing significantly to the spacecraft debris environment, while a non-catastrophic collision is one in which the primary object is largely intact, with minor additional generation of debris objects resulting from the collision. As such, consideration of the potential debris generation from a given event or the "consequence" of a prospective collision is an important addition to evaluating the risk a specific event poses to the orbital regime at which the potential collision may occur.

Considering the consequence of a prospective conjunction as it relates to the spacecraft operational environment is critical to maintaining a safe environment for current and future missions. This means that the risk posed by a potential conjunction to the space environment is a combination of two factors, the likelihood, or probability of collision, and the possible, additional debris production from a specific collision. Current practices in assessing collision risk are focused almost exclusively on the probability of collision aspect, whereas the use of an approach incorporating the consequences of a perspective collision allows for more informed decisions to be made with regard to the risk posed to the operational environment.

Taking into account the potential for debris object generation as a consequence of a prospective collision will allow operators to better tailor their collision risk management processes to preserve the space environment. If the potential for debris generation is not taken into consideration, future collisions may increase the debris object density in favorable satellite orbits and render them unusable because operators, faced with multiple serious conjunctions and able to remediate only a subset of them, may unwittingly choose to remediate only those with small debris production potential. Taking debris generation into account can also decelerate the onslaught of Kessler syndrome in the future [2]. This approach may be of considerable interest to satellite operators in geosynchronous orbit, where low velocity conjunctions are commonplace.

Previous work by Hejduk et al. [3] focused on developing methodologies to estimate debris generation for specific conjunctions and probability distribution functions of conjunction severity by orbit type based on historically observed conjunctions. These previous methods were dependent on a number of spacecraft estimation processes, and preliminary attempts at estimation were examined for accuracy against the RORSAT Coolant Spheres.

Current efforts contained in this paper are focused on refining the secondary object size estimation process using enhanced methodology, validating this process against known satellite objects, and presenting a readily readable method of interpreting these collision consequence results using this methodology. For validation purposes, a large number of NanoSats with known characteristics were compared to the secondary object size estimation process outlined in this paper.

\section{Debris Generation Methodology}

The NASA Orbital Debris Program Office (ODPO) has been studying the subject of collision and explosion-spawned space debris for the past several decades. Using information gleaned from known satellite collisions and staged hyperkinetic collisions, 
relationships have been developed to estimate the size and number of debris pieces that may be generated by a hyperkinetic impact. The relationships are contained as part of ODPO's EVOLVE 4 satellite break-up model [4].

The first consequence-based assessment of a potential conjunction is whether a conjunction, should it produce a collision, will result in a "catastrophic" collision, that is, a collision that produces widespread fragmentation of the primary object as well as the secondary; or "non-catastrophic," wherein only the secondary object is likely to fragment. This determination is made through a relationship based on the relative momentum of the two objects; a collision may be considered "catastrophic" if the relative momentum exceeds $40,000 \mathrm{~J} / \mathrm{kg}$.

$$
\frac{M_{s} V_{r e l}^{2}}{2 M_{p}}>40,000 \frac{\mathrm{J}}{\mathrm{kg}}
$$

Once the "catastrophic" or "non-catastrophic" nature of the event has been determined, an additional relation is used to estimate the possible number of debris objects that may be generated by such an event.

$$
N\left(L_{c}\right)=\left\{\begin{array}{r}
0.1\left(V_{\text {rel }} M_{s}\right)^{0.75} L_{c}^{-1.71}, \frac{M_{s} V_{r e l}^{2}}{2 M_{p}} \leq 40,000 \\
0.1\left(V_{\text {rel }}\left(M_{s}+M_{p}\right)\right)^{0.75} L_{c}^{-1.71}, \frac{M_{s} V_{r e l}^{2}}{2 M_{p}}>40,000
\end{array}\right.
$$

In the previous equations, $L_{c}$ refers to the characteristic length of the debris piece size threshold above which the operator is concerned, i.e., how many pieces will be generated with a characteristic length larger than $L_{c}$. A reasonable value for this variable would be the minimum characteristic length for which an operator might reasonably expect tracking data, such as $5 \mathrm{~cm}$ for the perceived tracking fidelity of the Space Fence.

The previous equations are relatively straightforward to evaluate if all terms are known; however, for prospective conjunctions, often times the secondary mass values are not known as the secondary objects involved in conjunctions are frequently debris objects such as those from the Fengyun or Iridium-COSMOS debris generation events. There are many possible reasons why the secondary object characteristics may be unknown; this is simply the most dominant one. The relative velocity of the two objects is generally fairly well known due to orbit determination processes for the two objects, as is the primary object's mass which is known from the spacecraft's operator, but characteristic length and mass of the secondary object are still unknown.

\section{Unknown Satellite Mass Estimation Process}

A method presents itself as convenient for estimating the secondary object's mass at lower altitudes using parameters contained within the drag equation:

$$
a_{\text {drag }}=-0.5 \frac{C_{D} A}{M} \rho\|\vec{v}\| \vec{v}
$$

Estimation of an object's drag characteristics is of significant import in the orbit determination process, as this is the primary non-conservative force acting on objects in low earth orbit, where most spacecraft missions also operate. As such, the collective terms for satellite drag can be determined as part of the orbit determination process and collected as a term known as the ballistic coefficient and is typically included in reported spacecraft states.

$$
B C=C_{d} A / M
$$

Knowledge of the ballistic coefficient allows for estimation of the object mass, but presents a new problem, namely that the drag coefficient $\left(C_{d}\right)$ and frontal area $(A)$ of the spacecraft must then also be estimated or known. In this study methods to estimate reasonable drag coefficients and frontal areas from known satellite quantities are proposed and validated by examining the characteristics of known NanoSats, and as these objects are known, their drag coefficients can be estimated using a cuboid drag model as proposed by Walker et al. [5]. The cuboid drag model is broadly applicable regardless of object type for nanosatellites as they typically conform to the cuboid model, though there may be more profound variation due to nanosatellite orientation. This allows for a preliminary examination of model accuracy for when the object profile is relatively known, though operationally the 
most calamitous estimation of $C_{d}$ may be used as a conservative estimate of the possible catastrophic nature of a collision. For this analysis the nanosatellite drag coefficients are generally presumed to be in line with the more oblong configurations, which show greater variation than strict cube-shaped approximations. Assuming an oblong cuboid satellite form, the drag values can be roughly approximated from Figure 8 of Walker et al. [5] as linearly varying based on exospheric temperature, which can be estimated by a given atmospheric model of choice.

$$
C_{d}=2.4+\frac{0.6}{800}\left(T_{i n f}-200\right)
$$

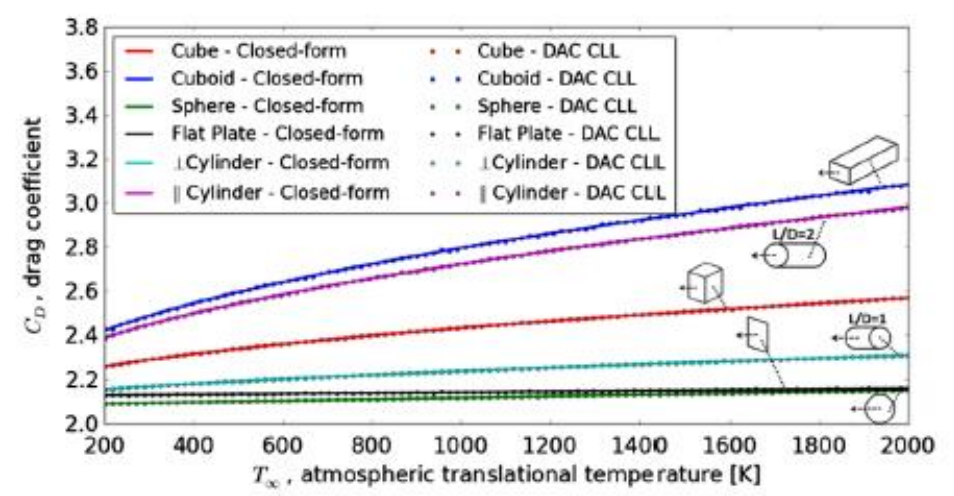

a)

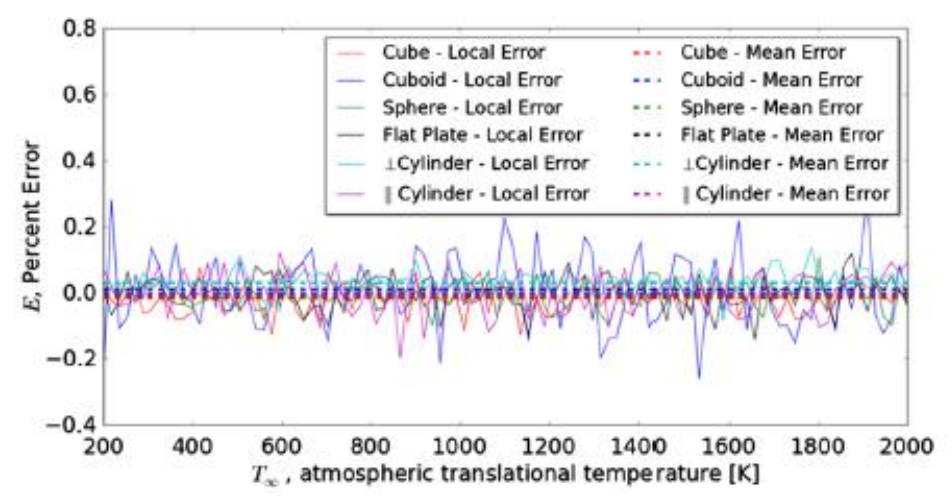

b)

Fig. 8 a) $C_{D}$ as a function of the atmospheric translational temperature $T_{\infty}$, for six different closed-form solutions and b) comparison of the percent error $E$, between the closed-form and DAC solutions.

Fig. 1. Recreation of Figure 8 from Walker et al. [5] denoting drag coefficient dependence on exospheric temperature.

While there are certainly variations in the drag coefficient as estimated, these have been shown to often be overshadowed by frontal area variations and atmospheric density variations, particularly at lower altitudes as shown in Pilinski et al. [6], which estimated that $C_{d}$ variations are typically only on the order of $2 \%$ except in extremely low altitude cases. For each determined best estimate of the drag coefficient, a number of samples are conservatively drawn using a normal distribution with a relative standard deviation of 5\% (significantly larger than Pilinski's stated 2\%) and a mean as determined above, using a sampling methodology allows for a predicted distribution of collision consequence. This larger sigma value is used so that a larger spread of $C_{d}$ values may be examined for their effect on a collision. 


\section{Unknown Satellite Frontal Area Estimation Process}

The frontal area of the secondary object may be estimated using a relationship between the presented area of the satellite and the object's Radar Cross Section (RCS). The intensity of the return of radar observations used in the orbit determination of an unknown object is characterized as the RCS and is a solved-for variable in the radar range equation if the Signal to Noise Ratio (SNR) and range are known. Typically this is reported as the median RCS value, as the RCS distribution does not conform to a normal distribution. If the wavelength of the observing radar station is known, the characteristic length of the object may be estimated using the median RCS (a value that is made available from the 18 SPCS, for example). This singular value is not necessarily an accurate representation of the spacecraft characteristic length and is better characterized as a distribution, the NASA ODPO model was developed in order to take a distribution of RCS values and produce a distribution of characteristic lengths. This study approximates the distribution of RCS values as a Swerling III, grounded by a single shape parameter that can be derived from the RCS median value. While this methodology is not an ideal manner of characterizing RCS distributions, it outperforms most other choices, as presented by Hejduk and DePalma [7].

This approach improves upon previous work, which used a less robust method of characterizing the RCS distribution that separated the RCS distribution into a sum of two distributions. Using this Swerling III distribution, a large set of samples representing the secondary object RCS are generated for use in the mass estimation process.

While this RCS value has units of area, it is not usually indicative of the satellite cross sectional area, and hence must be translated to an effective frontal area. The ODPO has examined this in prior research and developed a size estimation model (SEM), which was developed by fragmenting a satellite in a vacuum, measuring the characteristic dimensions of the resulting fragments, and then evaluating said pieces' radar returns when illuminated in all possible configurations [4]. Radar theory allows a dimensionless relationship to be established between an object's characteristic length (normalized by the radar wavelength) and the object's RCS (normalized by the square of the radar wavelength). This process provides a method by which the characteristic length may be determined if the RCS and the tracking radar's wavelength or frequency is known; if not, a good approximation of a generic radar may be established using radar frequencies of either UHF $(\sim 430 \mathrm{MHz})$ or L-Band $(\sim 1200 \mathrm{MHz})$, depending on object RCS value. The characteristic length distribution of the secondary object is then used to approximate a distribution of estimates of the satellite frontal area:

$$
A=\frac{\pi L_{c}^{2}}{4}
$$

\section{Satellite Size Validation Method}

At this point a method has been established by which an object's mass may be estimated using readily available data from the orbit determination process. To validate this approach a large set of NanoSats for a range of operational altitudes were examined. The initial set of examined satellites was comprised of roughly 1000 NanoSats. This set was then investigated to determine their prescribed masses, dimensions, satellite bus size, and Satellite ID numbers. The initial set of 1000 NanoSats was reduced to 530 satellites based on satellite specification availability and launch successes. As all of these spacecraft are cuboid in nature due to the nature of satellite buses available at that size, the expected frontal area for each spacecraft was estimated as the mean area presented by the spacecraft assuming a random tumbling nature. This estimation assumes a sinusoidal behavior as it relates to the presented satellite area, which makes the estimated area an average between the maximum and minimum presented areas. Using this approach, an independent data set of small object masses and frontal areas had been established. For an irregularly shaped object, a formula proposed in the NASA standard breakup model [4] and expounded upon by Hanada [8] for the average crosssectional area is:

$$
A_{\text {total }}=\frac{\left(A_{x y}+A_{y z}+A_{x z}\right)}{3}
$$

As the spacecraft in question are cuboid, the maximum triplet dimensions of $x, y$, and $z$ can be determined in a relatively straightforward manner as the outer dimensions of the cuboid are known. 
Next, orbit determination data was retrieved from relevant Conjunction Data Messages (CDMs) pertaining to these objects, including orbit regime, RCS, and ballistic coefficient. This exercise further reduced the available Nanosat population for validation to 371 based on CDM data availability.

\section{Validation Results}

Combining the previous sections' analyses and processes yields a distribution of object frontal areas and masses of the prescribed NanoSats. For each spacecraft 10,000 samples were derived, taking random draws of ballistic coefficient and frontal area as estimated from the RCS distribution. For validation purposes, the median values of frontal area and mass estimates are taken as the best estimate of the object's characteristics from this process and the quartile values are taken as the range of the uncertainty in this estimate. The estimated masses and frontal areas of these NanoSatellites were then compared with the independently determined values from the spacecraft specifications as researched, and compared in a normalized ratio sense. The distribution of these ratios is summarized below:

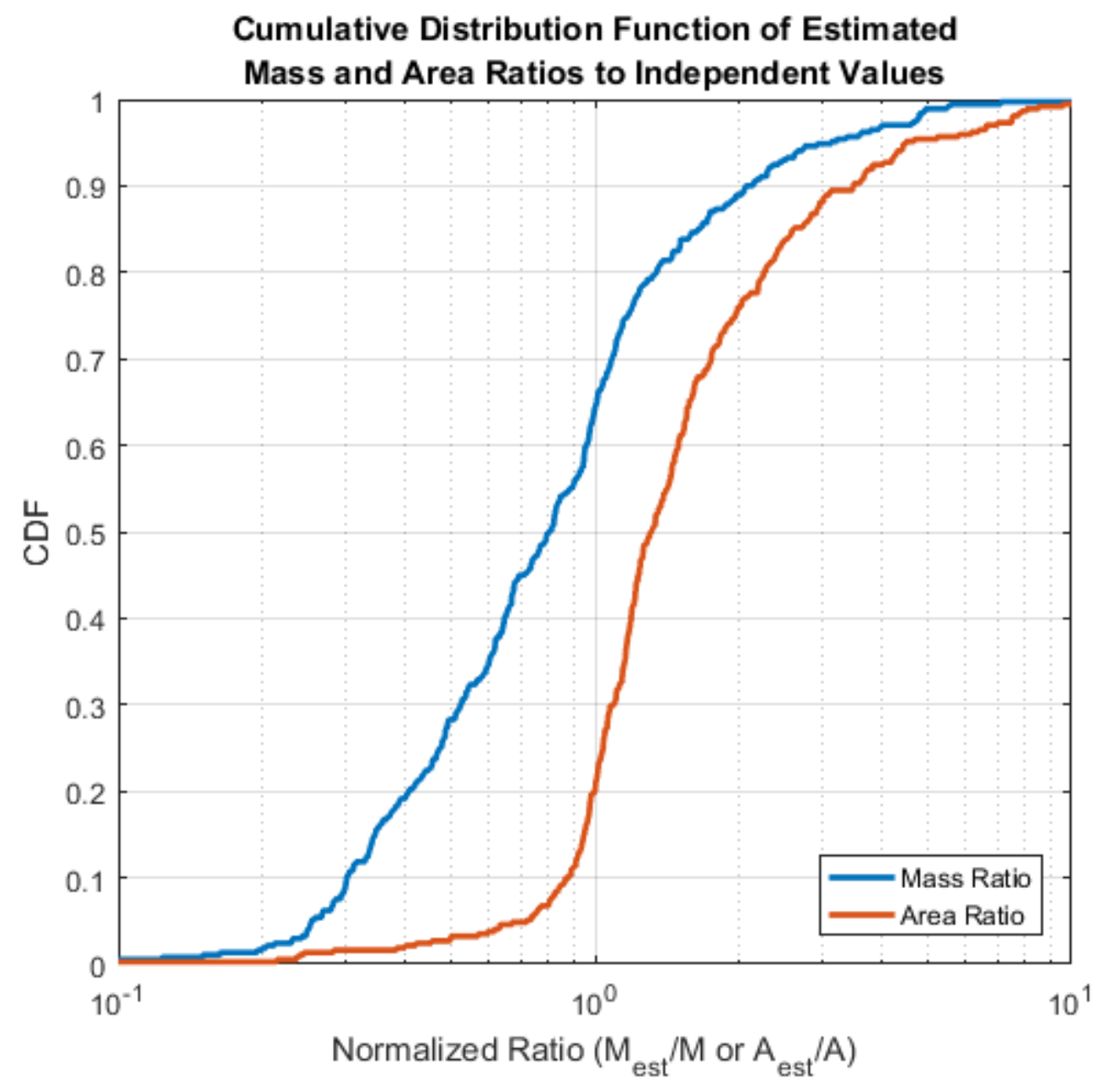

Fig. 2. Cumulative Distribution Function of Estimated Parameter Ratios to Researched Values for 371 NanoSats

Figure 2 shows the cumulative distribution of estimated parameter ratios and good general agreement for both area and mass ratios for described NanoSats. This figure shows that the frontal area estimates for the satellites in question typically overestimate the actual value, while the mass estimates tend to underestimate the actual vale. The mass estimates show the following ranges of accuracies for desired confidence intervals using this methodology: 
- Mass Estimate Accuracy Ratios for NanoSatellites Show the Following Range at 68\% Confidence: 0.353 - 1.574

- Mass Estimate Accuracy Ratios for NanoSatellites Show the Following Range at 95\% Confidence: 0.227 - 4.709

These confidence intervals were selected to mirror the standard deviations of a normal distribution (1-sigma, 2-sigma), and the mass estimates show a $68 \%$ confidence that the mass estimates of the satellites will be roughly within $60 \%$ of the actual spacecraft mass, and a 95\% confidence that the mass estimate will be within a factor of 5 of the actual mass estimate. In this process, the satellite masses were overestimated $\sim 36 \%$ of the time. In order to err on the side of conservatism, users may opt in the future to artificially inflate the $C_{d}$ values (or add a multiplicative biasing coefficient), which in turn will inflate the secondary object mass estimates, leading to a higher probability that the prospective collision may be "catastrophic".

\section{Collision Consequence Assessment for Individual Events}

The end goal of these sets of analysis is to provide satellite operators with a method by which to assess possible consequences of a prospective collision. Useful information to be provided to the operators would be the probability of a prosepective conjunction being a "catastrophic" collision, the expected number of debris objects generated for both "catastrophic" and "noncatastrophic" events, and the distribution of the number of expected debris pieces based on a large number of trials. Using the validated methodology outlined above, a distribution of secondary object masses may be determined for a specific conjunction using a large sample number and the distributions outlined above. Using this distribution of secondary object masses as well as the characteristics of the prospective conjunction, a probability that the conjunction will be catastrophic may be assessed based on the number of mass estimates which result in a "catastrophic" collision assuming the two object's relative velocities and the primary object's mass are well known. By distinguishing which members of the secondary mass estimate distribution are catastrophic and which are not, the expected number of generated debris pieces for a given conjunction may be assessed for both the "catastrophic" and "non-catastrophic" cases. The two values for the expected number of debris pieces can then be reported to give operators a better perspective on the possible debris generation given a specific conjunction. Below follows two examples of conjunctions and the prospective consequences of a collision.

The following figures are examples of prospective outputs for an event which have very low and very high likelihood, respectively of being catastrophic. In these cases 10,000 samples of secondary mass estimate were used for assessment purposes.
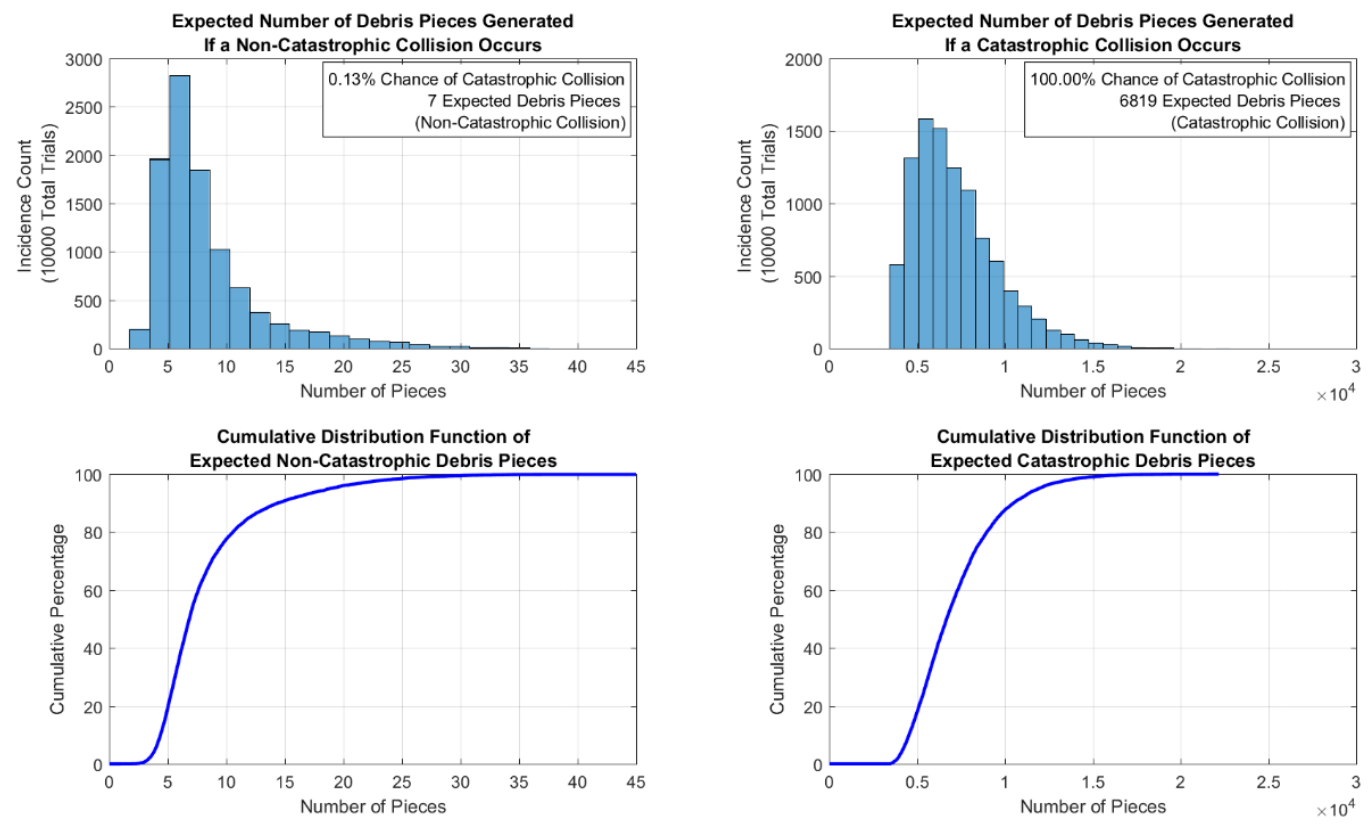

(a)

(b) 
Fig. 3. Collision Consequence Assessment for Independent Individual Events (a) Non-Catastrophic (b) Catastrophic

For many events, the probability that the prospective collision will be "catastrophic" or "non-catastrophic" is nearly certain. When this is the case, only the relevant chart is of interest. However, when this is not the case, the debris generation processes must be separated by trial on a "catastrophic"/"non-catastrophic" basis, and both sets of results presented. This is due to the radical difference in scale in prospective debris generation profiles for the two varieties of collision.

Above in Figure 1.a there is only a $0.13 \%$ chance the collision will be "catastrophic", with an expected number of generated debris pieces for the "non-catastrophic" case of: 7 debris pieces generated. However, for the few cases where the event was judged to be catastrophic, almost 2000 debris pieces were expected to be generated. In Figure 1.b, there is a >99.99\% chance the prospective collision will be "catastrophic", as no trials resulted in "non-catastrophic" cases, with an expected number of debris pieces equal to 6819 .

\section{Conclusions and Future Work}

This study presents steps forward in the estimation of the mass and size of secondary objects which pose a collision risk to operational spacecraft, but whose characteristics are not widely known. These estimation processes are validated against a set of known NanoSats, and show acceptable agreement, but are far from perfect in the estimation of these satellite states. These updated methodologies for assessing secondary object mass are used in a collision consequence assessment to provide operators with additional information regarding the potential for detrimental effects on the operational space environment through the likelihood of debris generation events, and the expected amount of debris articles generated from such an event. A method is then proposed for presenting relevant information to spacecraft operators. In future work, better drag coefficient estimation methodologies may be explored, mass estimates may be inflated to err on the side of conservatism, and additional characteristics of the predicted debris pieces may be assessed. One such additional characteristic may be the orbital half-life of the debris field based on drag modelling and atmospheric density predictions.

\section{References}

[1] Kaplan, S. and Garrick, B.: "On the Quantitative Definition of Risk." Risk Analysis, Vol. 1 No. 1 (1981), pp. 11-27.

[2] Kessler, D.J. and Cour-Palais, B.C.: "Collision Frequency of Artificial Satellites: The Creation of a Debris Belt." Journal of Geophysical Research 83 (1978), pp. 2637-2646.

[3] Hejduk, M., Laporte, F., Moury, M., Kelso, T.S., Newman, L., Shepperd, R. "Consideration of Collision "Consequence” in Satellite Conjunction Assessment and Risk Analysis, International Symposium on Space Flight Dynamics, Matsuyama, Japan, 2017.

[4] Johnson, N.L., Krisko, P.H., Liou, J.-C., and Anz-Meador, P.D.: "NASA’s New Breakup Model of EVOLVE 4.0." Advances in Space Research, Vol. 28 No. 9 (2001), pp. 1377-1384.

[5] Walker, A., Mehta, P., Koller, J., "Drag Coefficient Model Using Cercignani-Lampis-Lord Gas-Surface Interaction Model”, Journal of Spacecraft and Rockets, Vol. 51, No. 5 (2014), pp. 1544-1563.

[6] Pilinski, M., Argrow, B., Palo, S., "Semi-Empirical Satellite Accommodation Model for Spherical and Randomly Tumbling Objects", Journal of Spacecraft and Rockets, Vol. 50, No. 3 (2013), pp. 556-571.

[7] Hejduk, M., DePalma, D., "Comprehensive Radar Cross-Section "Target Typing” Investigation for Spacecraft", Advances in the Astronautical Sciences, Vol. 135, 2010, AAS 09-301.

[8] Hanada, T., Liou, J. C., Krisko, P., Nakajima, T., "For Better Calculation of the Average Cross-Sectional Area of Breakup Fragments", Transactions of Space Technology Japan, Vol. 7, Iss. 26, 2009, pp. 25-30. 